\title{
Baksan Air Shower Array: new prospects for old facility
}

\author{
Alexander S. Lidvansky ${ }^{1, *}$ \\ ${ }^{1}$ Institute for Nuclear Research, Russian Academy of Sciences, Moscow, Russia
}

\begin{abstract}
The Carpet air shower array of the Baksan Neutrino Observatory is in operation for a long time, and it was modernized more than once. A short review of important results obtained with this array is given, as well as future prospects, since at the moment the new project called Carpet- 3 is in progress, using the old array as its basis. The purpose of the new project is to substantially increase the muon detector area (quite soon up to $400 \mathrm{~m}^{2}$ and later up to $600 \mathrm{~m}^{2}$ ). This improvement will allow one to reach a very good sensitivity to diffuse cosmic photons by selecting muon-poor showers. The energy range where the new experiment will be competitive with other experiments of gamma-ray astronomy is near and below $100 \mathrm{TeV}$.
\end{abstract}

\section{Introduction}

The Carpet air shower array [1, 2], sometimes also called BASA (Baksan Air Shower Array) was the first large facility of the Baksan Neutrino Observatory (Fig. 1). Its main part, a carpet of 400 scintillators with a total area of $200 \mathrm{~m}^{2}$ represented the model of a single plane of the future Baksan Underground Scintillation Telescope (BUST), then under construction. In spite of such an applied purpose of its creation, the Carpet array appeared to be an instrument for cosmic ray studies with enormous significance of its own. Originally, the central scintillation Carpet was surrounded by six outdoor huts, each of which containing 18 standard scintillation detectors and a set of Geiger counters for calibration purposes (the laboratory, headed by G.B. Khristiansen, from the Nuclear Physics Institute of Moscow State University participated in installing the huts and equipping them). Later, this original set was supplemented with a neutron monitor and a muon detector. The Carpet array was modernized more than once, and is still in operation at the present time. Moreover, at the moment the new project Carpet- 3 is under realization on its basis: it was demonstrated that with a radical increase of the muon detector area, due to selection of muon-poor showers, the array can have the world-best sensitivity to primary cosmic gamma rays with energies in the range 10-100 TeV [3]. In view of this future development of the array, it is worthwhile to review the most interesting previous results obtained during its long history. Some of them are very far from typical cosmic ray physics made with air shower arrays.

\section{EAS studies}

The first experiments with the Carpet facility were dedicated to studies of the lateral distribution function (LDF)

\footnotetext{
*e-mail: lidvansk@lebedev.ru
}

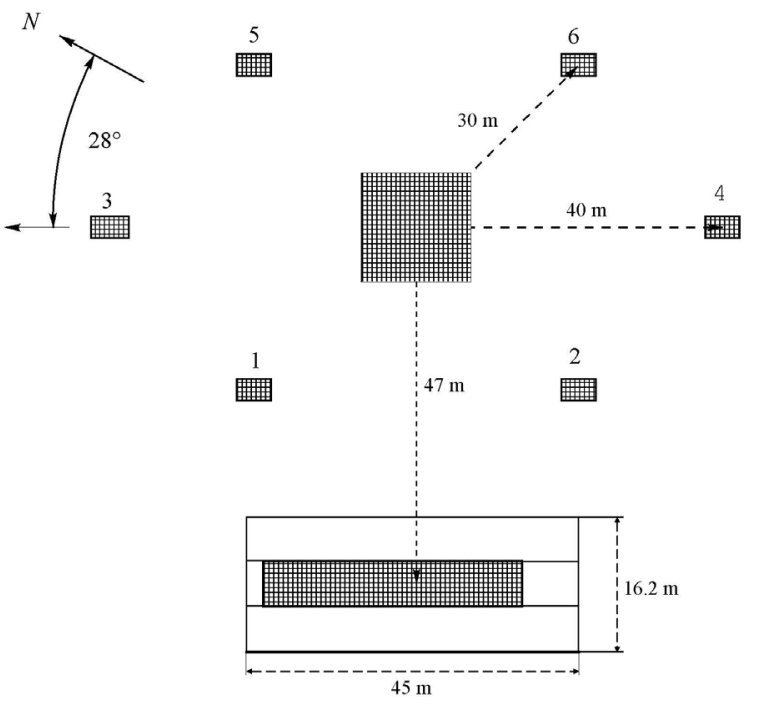

Figure 1. The layout of the Carpet air shower array in its original form with the first part of the Muon Detecor constructed later.

of air shower particles. Owing to a large continuous area with fine structure the shower axes were detected with a good accuracy, while large area remote scintillation detectors also measured the particle density with high accuracy. These two factors allowed really high-precision measurements of LDF to be made, though in a limited range of distances from the shower axis $[4,5]$. The same unprecedented accuracy allowed us to discover anomalous showers with sharp fluctuations of LDF [6].

\section{High transverse momenta in hadron-hadron interactions}

Next, the large area of the Carpet has made it possible to investigate the spatial structure of EAS [7] near the axis 


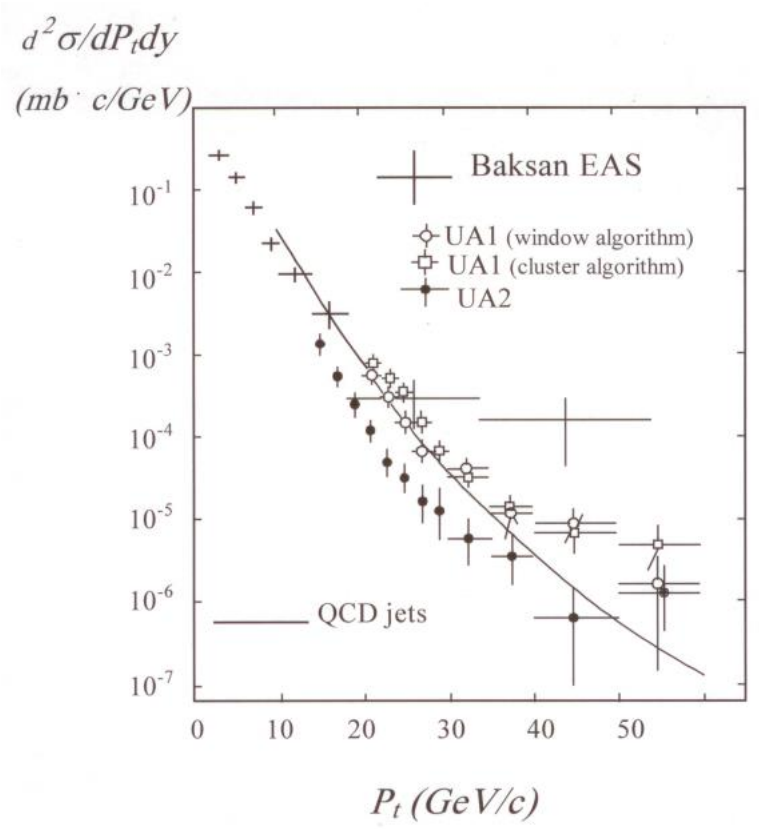

Figure 2. Cross-section of high- $\mathrm{p}_{t}$ events in an air shower expriment (1981) compared to a calculation for QCD jets and first experiments at the CERN SPS collider (1982)

and to study multi-core showers $[8,9]$. By interpreting sub-cores in multi-core air showers as a result of generation by leading particles of high-transverse-momentum jets, we succeeded in deriving the cross-section of generation of such jets at an energy whose value was estimated as $500 \mathrm{GeV}$ in the center of mass system. At that time this cross section was already calculated in the quantum chromodynamics approach for $540 \mathrm{GeV}$ - the planned energy of the SPS-collider, then under construction. In [10] it was demonstrated that the cross-section of high- $\mathrm{p}_{t}$ jet production, derived from the analysis of multi-core showers detected by the Carpet array, was in good agreement with predictions of quantum chromodynamics. When the CERN SPS-collider was put into operation, the same result was obtained by the UA1 and UA 2 collaborations, but this was done some year and a half later (Fig. 2).

\section{Gamma-ray astronomy}

In 1980s a deluge of works, both experimental and theoretical, on ultra-high energy gamma ray astronomy was stimulated by one publication (obviously wrong, as it became clear much later) of the Kiel University group that pretended to observe a signal from X-ray source Cygnus $\mathrm{X}-3$ at energies higher than $10^{15} \mathrm{eV}$. The Carpet participated in this campaign too. However, this noisy activity finally gave almost no positive results, with perhaps a few exceptions. Some announced detections had small statistical significance and were not confirmed later. The BASA obtained only an upper limit on the permanent gamma-ray flux from Cygnus X-3 at $10^{13} \mathrm{eV}$, but one burst-like event lasting for three days was detected [11] from this source. This burst had happened on September 14-16, 1985, a couple of days later than a very strong (the strongest for all

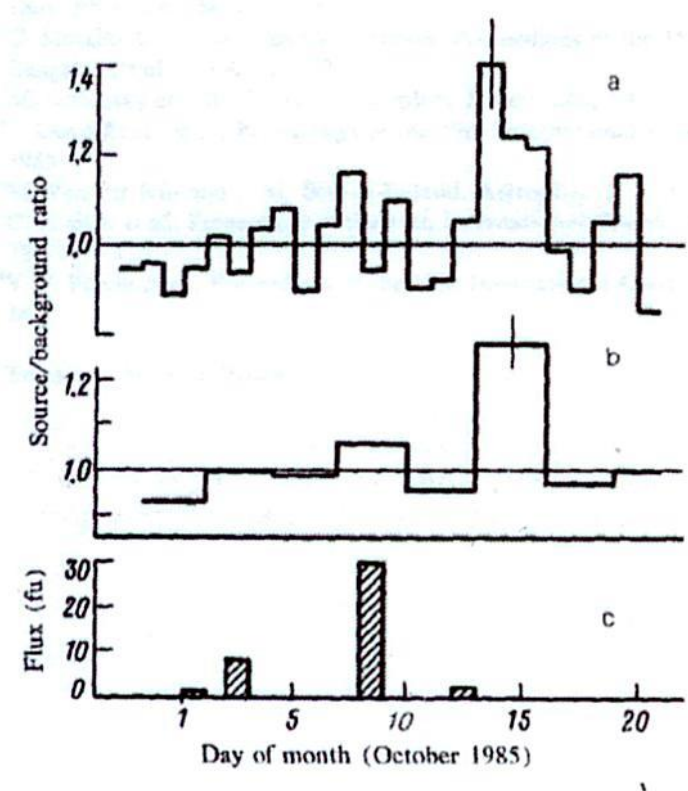

Figure 3. (a) Ratio of daily count rates in the Cygnus X-3 angular cell to the average count rate; (b) the same, average over 3 days; (c) spectral power density in the radio burst in flux units (Jy).

observation time) outburst of radio emission from Cygnus X-3 (see Fig. 3 taken from [12]). Also, a shorter burst was recorded from another source.

In [13] the Baksan group announced that a possible burst had occurred in the Crab Nebula at an energy of 100 TeV on February 23, 1989.

The Kolar Gold Field (KGF) group in India confirmed this result [14], and in a final publication [15] the data of two more groups were presented, Tien Shan (then Soviet Union) and EAS-TOP (Italy), who also confirmed, though with a small statistical significance, the existence of the effect.

It is understandable that the results of [15] were met with some distrust: at that time a burst of such a high energy (hundreds of $\mathrm{TeV}$ ) seemed too exotic, especially from the source, which, though it had just been recorded by Cherenkov telescopes at $\mathrm{TeV}$ energies, served as a pattern of stability (so that for a long time it was considered as the "standard candle" in very high energy astronomy and in X-ray astronomy). However, many years later the flares from the Crab nebula were detected by satellite gammaray telescopes, AGILE and FermiLAT, at energies of a few hundred MeV. As was demonstrated in [16], one of the AGILE flares [17] had the time structure fully similar to that of the burst on February 23, 1989 (accounting for a single scale factor). This fact compels one to take the latter most seriously: after all, this signal from a celestial source has been recorded at a highest energy ever detected.

\section{Variations of cosmic rays}

The large area of detectors suggests high count rates, which allows one to study variations of cosmic rays with a good statistical accuracy. By measuring the daily wave of 
intensity in sidereal time the anisotropy of galactic cosmic rays was investigated with three $\mathrm{BNO}$ facilities at three different energies: BUST (2.5 TeV), Carpet $(10 \mathrm{TeV})$, and Andyrchi $(100 \mathrm{TeV})[18,19]$. In [20] the true direction of the cosmic ray anisotropy vector was reconstructed using the data of two of the last arrays.

The most interesting results were obtained studying variations of different components of secondary cosmic rays during thunderstorms.

Variations of secondary cosmic rays during thunderstorms were first proved to be related to the strong electric field (measured near the ground surface) in a pioneering experiment carried out by A.E. Chudakov with collaborators in early 1980s (for references see [21]. The new version of the same experiment $[22,23]$ yielded a lot of data on variations of cosmic rays during thunderstorms. Two types of experimental data were obtained: (1) regular variations of the counting rates of the soft and hard components correlated with the near-earth electric field of the atmosphere during thunderstorms and (2) bright events or strong changes in the counting rate of these components. Figure 4 presents as an example one event that illustrates basic features of the bright events. First the soft component gives only increases of intensity.

Much later similar events were detected at Mt. Aragats in Armenia and were referred to by A. Chilingarian as TGEs (thunderstorm ground enhancements) [24]. Most disturbances of the muon intensity are negative, though some positive effects are also possible, as one can see in Fig. 4. The analysis of experimental data allowed us to interpret TGE as a result of cyclic generation of particles in a strong electric field [25]. Due to acceleration of runaway electrons and positrons in opposite directions in the strongly scattering medium (air), a feedback loop arises, thus resulting in exponential increase of particle intensity in a limited volume, where the electric field exceeds the critical strength. Gamma rays from the acceleration region make the main contribution to TGE count rate. Further analysis [26] has shown that the altitudes (and, accordingly, critical fields) of these acceleration regions can be quite different, even when TGEs look alike. Sometimes, the height of acceleration region is rather low (3-5 $\mathrm{km}$ above the observation level). These events are brighter (up to $30 \%$ of intensity in the energy range $10-30 \mathrm{MeV}$ ) and show no effect in the flux of muons. In other cases the height of generation is estimated to be in the stratosphere, and these events (with 3-5\% TGE amplitudes) are obviously connected with disturbances of the intensity of muons with an energy threshold equal to $100 \mathrm{MeV}$ (in fact, variations of the muon intensity have been investigated at different energy thresholds, but the effect is the strongest for the lowest threshold). Both signs (increase and decrease) of such disturbances with amplitudes of no more than $1 \%$ are possible, though the negative disturbances are more frequent. In some cases the TGEs with muon effects can coincide with geomagnetic pulsations [27] (the high-precision measurements of the geomagnetic field are performed in deep underground rooms of the Baksan Neutrino Observatory, $1 \mathrm{~km}$ apart from the BASA). Since both muon disturbances and geomagnetic pulsations

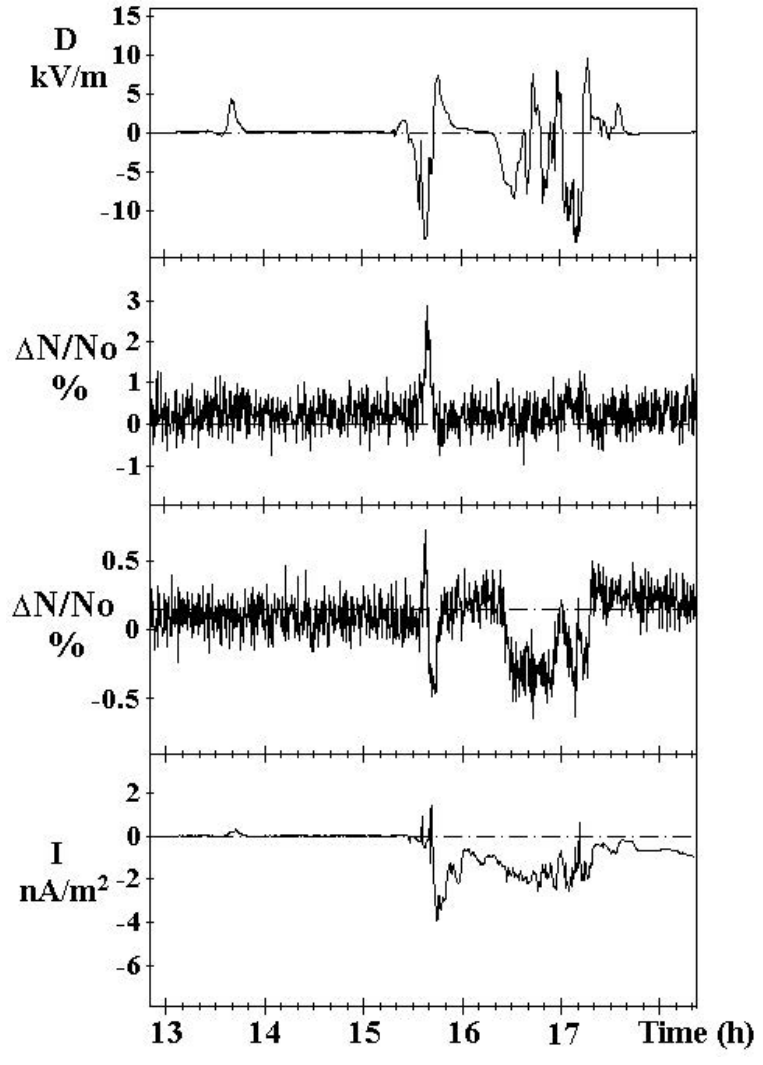

Figure 4. A thunderstorm event on September 24, 2000 recorded at the Baksan Valley, North Caucusus. From top to bottom: 1. The near-earth electric field strength during the thunderstorm. 2. The count rate of the soft component of cosmic rays (electrons and gamma-rays within the range $10-30 \mathrm{MeV}$ ) as measured by uncovered scintillators (108 detectors, $30 \mathrm{~cm}$ thick filled with liquid scintillator, total area $54 \mathrm{~m}^{2}$. 3. Counting rate of the hard component of cosmic rays (muons with energies above 100 $\mathrm{MeV}$ ) measured by 400 scintillators with a total area of $200 \mathrm{~m}^{2}$ under a concrete roof $21 \mathrm{~g} / \mathrm{cm}^{2}$ thick. 4. Precipitation electric current.

have rather long duration (minutes or tens of minutes) a hypothesis of the existence of a new type of high-altitude discharge (analog of glow discharge, but produced by relativistic electrons) was put forward. In order to verify this hypothesis, remote video cameras were installed (75 $\mathrm{km}$ from the BASA) to observe the region above thunderstorms. Though the sought-for events are very rare (observation is possible only for night-time thunderstorms on moonless nights) the glow was indeed detected [28, 29]. But as usual, the phenomenon appeared to be much more rich and complicated than expected. These experiments and their analysis continue at the moment, this line of research being very promising for discovering fundamental role played by cosmic rays in a thunderstorm atmosphere.

\section{Back to gamma-ray astronomy: the Carpet-3 project}

New possibilities for BASA have appeared after sensational publications of the IceCube results on detection of high-energy astrophysical neutrinos. The neutrinos produced in decays of charged pions should be accompa- 


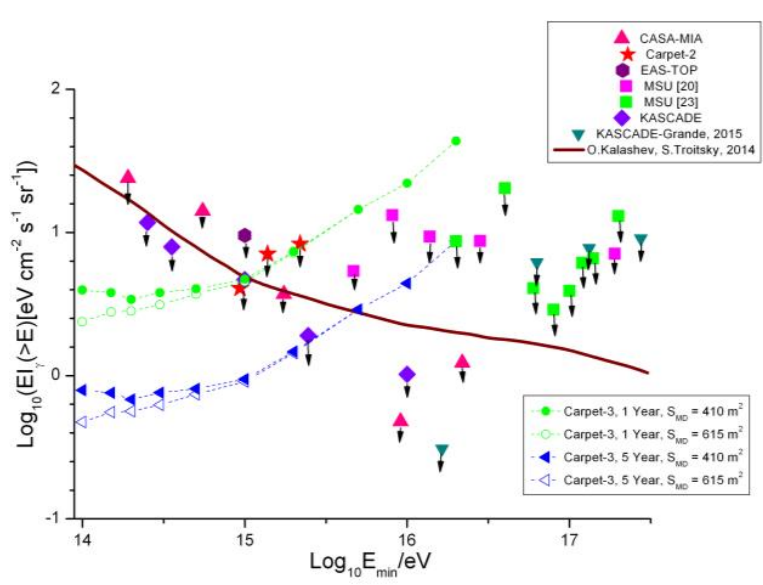

Figure 5. Limits on the integral flux of gamma rays versus their energy. Some existing upper limits are shown together with expectations for the Carpet-3 array

nied by gamma rays produced in decays of neutral pions. Hence, there is a motivation for new specialized experiments with more precise measurements of the flux of diffuse gamma rays at energies higher than $100 \mathrm{TeV}$.

Realization of the experiment suggests that continuous area of Muon Detector (MD) should be increased at first up to $410 \mathrm{~m}^{2}$ and then up to $615 \mathrm{~m}^{2}$. Also, 20 additional shower detectors will be installed to extend the array. Each such detector will contain 9 scintillation counters of $1 \mathrm{~m}^{2}$ area each. At the present stage 410 scintillation counters with total continuous area $410 \mathrm{~m}^{2}$ are installed in the MD underground tunnels, and they are totally equipped with electronics. The work on adjusting the electronics of scintillation counters and on constructing data acquisition system for the given configuration of $\mathrm{MD}$ is in progress. The efficiency of selection of gamma ray showers and the array sensitivity to their detection are calculated for different configurations of the array. Figure 5 shows the expected limits for the flux of cosmic diffuse gamma rays for two configurations of the Carpet-3 air shower array and for two values of data accumulation time. As is seen, even for the MD area of $410 \mathrm{~m}^{2}$ the new array will have the best sensitivity to the primary gamma ray flux with energies in the range $100 \mathrm{TeV}-1 \mathrm{PeV}$.

\section{Acknowledgements}

The work was partially supported by the Russian Foundation for Basic Research, grant no. 16-02-00687.

\section{References}

[1] E.N. Alexeyev et al., Bull. of the Russian Academy of Sciences: Physics, 38, no. 5, 1097-1100 (1974).

[2] E.N. Alexeyev et al., Bull. of the Russian Academy of Sciences: Physics, 40, no. 5, 994-997 (1976).
[3] D.D. Dzhappuev et al., Journal of Physics: Conference Series, 934, 012022 (2017).

[4] E.N. Alexeyev et al., Proc. 15th ICRC, Plovdiv, Bulgaria, 8, 52 (1977).

[5] E.N. Alexeyev et al., Bull. of the Russian Academy of Sciences: Physics, 42, no. 7, 1417-1419 (1978).

[6] D.D. Dzhappuev et al., Yadernaya Fizika, 29, no. 4, 957961 (1979).

[7] E.N. Alexeyev et al., Bull. of the Russian Academy of Sciences: Physics, 42, no. 7, 1240-1242 (1978).

[8] A.E. Chudakov et al., Proc. of 16th ICRC, Kyoto, Japan, 8, 222 (1979).

[9] D.D. Dzhappuev et al., Bull. of the Russian Academy of Sciences: Physics, 45, no. 7, 1239-1242 (1981).

[10] A. E. Chudakov et al., Proc. of 17th ICRC, Paris, France, 6, 183-186 (1981).

[11] V.V. Alexeenko et al., Il Nuovo Cimento, 10C, 151-161 (1987).

[12] V.V. Alexeenko et al., JETP Lett., 44, 202-205 (1986).

[13] V.V. Alexeenko et al., Proceed. of Intern. Workshop on Very High Energy Gamma Ray Astronomy, Crimea, 1989, ed. by A.A. Stepanyan, D.J. Fegan and M.F. Cawley, p. 137.

[14] B.S. Acharya et al., Nature, 347, 364-5 (1990).

[15] V.V. Alexeenko et al., Journ. of Phys. G: Nucl. Part. Phys., 18, L83-L88 (1992).

[16] A.S. Lidvansky, 35th ICRC, 10-20 July, 2017, Bexco, Busan, Korea. PoS (ICRC2017) 726.

[17] E. Striani et al., Astrophys. Journ., 765, 52 (2013).

[18] V.V. Alexeenko et al., Bull. of the Russian Academy of Sciences: Physics, 46, no. 9, 1726-1728 (1982).

[19] V.A. Kozyarivsky et al., Bull. of the Russian Academy of Sciences: Physics, 68, no. 11, 1596-1598 (2004).

[20] D.D. Dzhappuev et al., Astronomy Letters, 36, no. 6, 439444 (2010).

[21] A.S. Lidvansky, J. Phys. G: Nucl. Part. Phys., 29, 925-937 (2003).

[22] V.V. Alexeenko, N.S. Khaerdinov, A.S. Lidvansky and V. B. Petkov, Physics Letters A, 301, issues 3-4, 299-306 (2002).

[23] N.S. Khaerdinov, A.S. Lidvansky, and V.B. Petkov, Atmospheric Research, 76, issues 1-4, 346-354 (2005).

[24] A. Chilingarian, Journal of Physics: Conference Series, 409, 012019 (2013).

[25] A. S. Lidvansky and N. S. Khaerdinov, Bull. of the Russian Academy of Sciences: Physics, 71, no. 7, 1032-1034 (2007).

[26] N. S. Khaerdinov and A. S. Lidvansky, Journal of Physics: Conference Series, 409, 012225 (2013).

[27] K. Kh. Kanonidi et al., Astrophysics and Space Science Transactions (ASTRA), 7, 279-282 (2011).

[28] K. Kh. Kanonidi et al., Bull. of the Russian Academy of Sciences. Physics, 79, no. 5, 676-678 (2015).

[29] K. Kh. Kanonidi et al., Bull. of the Russian Academy of Sciences. Physics, 79, no. 5, 679-681 (2015).

[30] D D Dzhappuev et al., Journal of Physics: Conference Series, 934, 012022 (2017). 